\title{
Atomic spectra calculations for fusion plasma engineering using a solvable model potential
}

\author{
E. Charro and L.M. Nieto
}

Dedicated to Prof. N.H. March on the occasion of his 90 th birthday.

\begin{abstract}
The analysis of the atomic spectra emitted by highly ionized atoms is a field of extraordinary richness and a part of atomic physics with applications in astrophysics, engineering, fusion plasma and materials research. Certain elements have attracted considerable attention because they are useful for spectroscopic diagnostics in fusion plasmas, where a prediction of the experimental spectra is required. Taking into account this fact, the Relativistic Quantum Defect Orbital method has been applied to calculate relevant atomic data, as transition rates for emission lines, in a high number of atoms and ions. This formalism, unlike sophisticated and costly self-consistent-field procedures, is a simple but reliable analytical method based on exactly solvable model potentials, a type of problems that always attracted Prof. March attention. The method has the great advantage of a low computational cost, which is not increased as the atomic system becomes heavier. In this work, a highlight of this method is presented, together with an overview of the main atomic data obtained using it, which are useful in engineering for fusion plasma diagnostic.
\end{abstract}

\section{Introduction}

Present-day magnetic fusion devices, especially tokamaks, can generate plasmas with electron temperatures near $10 \mathrm{keV}$, and future machines may even reach temperatures of $25 \mathrm{keV}$ or more. This means that they can produce ions with very high charge even from heavy elements. From the beginning, highly charged ions have

E. Charro

Faculty of Education, Univ. Valladolid, 47011 Valladolid, Spain, e-mail: echarro@ dce.uva.es

L.M. Nieto

Department of Theoretical Physics, Atomic Physics and Optics, and IMUVA, Univ. de Valladolid, 47011 Valladolid, Spain, e-mail: luismiguel.nieto.calzada@uva.es 
been an important component of magnetically confined plasmas, and their presence has been highly advantageous for both plasma diagnostics and basic atomic physics studies, and harmful for the operation of a given device, if present in large quantities. The good and bad properties of highly charged ions derive from the fact that they radiate when embedded in a sea of electrons. Partially ionized heavy elements radiate profusely, mostly in the extreme ultraviolet wavelength range, while ions stripped to a few electrons within a closed shell, radiate predominantly in the Xray range. This radiation can be used to diagnose the plasma conditions, such as the electron temperature, electron density, ion temperature, ion transport and diffusion, and bulk plasma motion. On the other hand, the radiation from highly charged ions contributes to the overall power loss of the plasma: if the plasma contains too many heavy ions, the associated radiative power loss can be severe and prevent ignition and burn. The studies of plasma physics are also among the wide range of research interests of Prof. N.H. March, as can be seen in the following references $[1,2,3,4,5,6,7,8,9]$.

Highly charged ions play a crucial role in magnetic fusion plasmas given that they are used for many diagnostic purposes in magnetic fusion research [10]. These plasmas are excellent sources for producing highly charged ions and plenty of radiation for learning their atomic properties. These studies include calibration of density diagnostics, X-ray production by charge exchange, line identifications and accurate wavelength measurements, and benchmark data for ionization balance calculations. Studies of magnetic fusion plasmas also consume a large amount of atomic data, especially in order to develop new spectral diagnostics. In this way, line identification has been a diagnostic necessity in fusion research in order to identify the impurities that are inadvertently released into the plasma as different heating scenarios are explored. Early work focused on the transition metals, such as titanium, chromium, iron, and nickel, as well as on noble gases that could be admixed to the plasma. Once the possibility was created to inject any type of material into the plasma via laser injection, spectra of other metals were also investigated [11].

However, it is now clear that under conditions which prevail in low-density laboratory tokamak plasmas (where collisional deexcitation of metastable states is rather slow, leading to buildup of population of metastable levels), forbidden transitions, i.e., electric quadrupole and magnetic dipole transitions, gain in intensity and can be used to infer information about plasma temperature and dynamics. Forbidden lines due to magnetic dipole and electric quadrupole transitions between fine-structure levels of the ground and lower lying excited configurations of highly ionised atoms have been used for diagnostic of laboratory plasmas related to fusion devices. For those lines, fusion specialists need accurate atomic data, such as radiative transition probabilities. To respond to this need, atomic physicists have put a great deal of effort to computing (and sometimes measure) the required data.

To carry out those calculations, a number of ab initio codes are available, some of them have proved to yield rather accurate transition probability data. However, most of them are highly time-consuming and often infested with convergence problems. As an alternative, semiempirical methods are widely recognized that, for this type of studies, is of a clear convenience given that combine reliability and simplicity. Most 
of the semiempirical methods currently employed, which are quite often derived from a modification of a hydrogenlike wave equantion, the various interactions are given a different weighting. Deviations from the Coulomb potential, often classified as "penetration" and "polarization" (depending on the degree of overlap between the active and passive electrons, in the context of electronic transitions) may, at least to a reasonable extent, be accounted for by a model Hamiltonian that contains a parameter related to the quantum defect. More specifically, the two above effects seem to be adequately described in the relativistic quantum defect orbital (RQDO) method [12,13], which has been reformulated for the calculation of atomic data for forbidden transitions [14].

The paper is organized as follows. In the next section we will give a brief overview of the main aspects of the RQDO method, followed by the extension of this formalism to E2 transitions in Section 3. In Section 4, we illustrate how the use of the systematic trend of the atomic data along an isoelectronic sequence allows to predict data for new ions and to analyse the influence of the relativistic effect for highly ionized atoms. Section 5 is dedicated to show the calculated spectra of some of the ions with most interest for plasma diagnostic in fusion devices. Some concluding remarks put the end to the paper.

\section{The Relativistic Quantum Defect Orbital Method}

The relativistic formulation of the quantum defect orbital formalism, as proposed by Karwowski and Martín $[12,13]$ was based on the decoupling of the Dirac secondorder equation, and the interpretation of the resulting solutions, that, unlike previous models based on the quantum defect, provides exact eigenfunctions of a model Hamiltonian. The resulting orbitals are also valid in the core region retaining approximate core-valence orthogonality.

For a Coulomb potential $V(r)=-Z / r$, after the elimination of the spin and angular variables, the relevant radial part of the Dirac equation reads as follows in atomic units (used throughout the whole paper)

$$
\left[-\frac{d^{2}}{d r^{2}}+\frac{s(s \pm 1)}{r^{2}}-\frac{2 Z\left(1+\alpha^{2} E\right)}{r}\right]{\phi_{k}}^{ \pm}=E\left(2+\alpha^{2} E\right) \phi_{k}{ }^{ \pm},
$$

where $\phi_{k}{ }^{+}$and $\phi_{k}{ }^{-}$are related to the small and large components of the Dirac wave functions, $E$ is the difference between the total and the rest energy of the electron, $k$ is the relativistic angular momentum quantum number

$$
k= \pm(j+1 / 2), \quad \text { where } \quad j=\ell \pm 1 / 2,
$$

with $\ell$ the orbital quantum number, $\alpha$ is the fine structure constant, and

$$
s=k \sqrt{1-\frac{\alpha^{2} Z^{2}}{k^{2}}} .
$$


Two interpretations may be given to the solutions of Eq. (1): first, they give us the two components of the relativistic wave function wich is solution of the corresponding Dirac equation; second, they give us two solutions (corresponding to $k$ and $-k$ ) of a single scalar equation for a scalar (quasirelativistic) wave function $\psi_{k}$. A quasirelativistic theory is not only considerably simpler than the relativistic one, but, most important, it closely resembles the Schrödinger formulation. As a consequence, one may rather easily implement the quasirelativistic formalism in the majority of the methods being developed for the nonrelativistic theory. Equation (1) is formally very similar to the radial hydrogenic equation and passes into it in a trivial manner in the nonrelativistic limit of $\alpha \rightarrow 0$.

The quantum defect orbitals (QDO) are solutions of the Schrödinger equation

$$
\left[-\frac{d^{2}}{d r^{2}}+\frac{\chi(\chi+1)}{r^{2}}-\frac{2 Z_{n e t}}{r}\right] \psi_{k}{ }^{Q D}=2 E^{Q D} \psi_{k} Q D
$$

where $Z_{\text {net }}$ is the nuclear charge on the active electron at large $r$ and

$$
\chi=\ell-\delta+c,
$$

being $\delta$ the so-called quantum defect, and $c$ an integer chosen to ensure the correct number of nodes and the normalization of the radial wave function. The eigenvalue $E^{Q D}$ in Eq. (4) depends only on the noninteger part of $\chi$, being independent on $c$. The quantum defect $\delta$ is empirically obtained from the following expression:

$$
E^{Q D}=E^{x}=-\frac{Z_{n e t}^{2}}{2(n-\delta)^{2}},
$$

where $E^{x}$ is the experimental energy and $n$ is the principal quantum number of the nonrelativistic theory. A straightforward observation of Eqs. (1) and (4) clearly prove that the formal mathematical structures of the QDO theory and the scalar relativistic theory are the same. This formal similarity $[12,13]$ allowed us to reinterpret the QDO theory so that it would account for the major part of the relativistic effects.

Analogously to the nonrelativistic case shown in Eq. (4), the relativistic quantum defect orbital (RQDO) equation is written as follows

$$
\left[-\frac{d^{2}}{d r^{2}}+\frac{\Lambda(\Lambda+1)}{r^{2}}-\frac{2 Z_{n e t}^{\prime}}{r}\right] \psi_{k}^{R D}=2 e^{R D} \psi_{k}^{R D},
$$

where $Z_{\text {net }}^{\prime}$ depends on quantities already defined

$$
Z_{\text {net }}^{\prime}=Z_{\text {net }}\left(1+\alpha^{2} E^{x}\right)
$$

and the parameter $\Lambda$ is such that

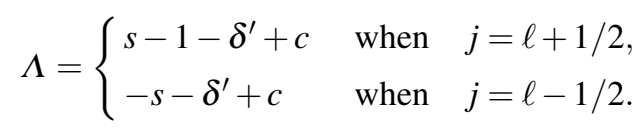


We have just introduced the so-called relativistic quantum defect $\delta^{\prime}$, a key element of the present method, which will be determined empirically from the experimental energy $E^{x}$ using the expression [13]

$$
-\frac{Z_{n e t}^{2}}{2\left(\eta-\delta^{\prime}\right)^{2}}=E^{x} \frac{1+\alpha^{2} E^{x} / 2}{\left(1+\alpha^{2} E^{x}\right)^{2}} .
$$

In the last equation the parameter $\eta$ is defined as

$$
\eta=n-|k|+|s|,
$$

in terms of quantities already introduced.

It should be stressed that this formulation is "exact" in the sense that it is equivalent to a four-component formulation based on the standard first-order form of the Dirac equation. All matrix elements, in particular the transition moments, may be expressed in a simple way, using the solutions of the second-order equation. A set of recurrent formulas which are fulfilled by the radial integrals [15] makes the formalism to be very simple and compact. Karwowski and Martín [12] have remarked that the relativistic density distribution approximates very well the exact one at large values of $r$. At small distances, the quality of the density deteriorates, as happens when the nonrelativistic QDO densities are compared with the exact nonrelativistic ones. Fortunately, the consequences of this drawback are very seldom reflected in the quality of the QDO and RQDO transition probabilities, because the strongest contribution to radial matrix elements comes, in most cases, from large radial distances.

The most important difference between the RQDO and QDO equations is the explicit dependence of the former on the total angular momentum quantum number $k$. As a consequence, values of the relativistic quantum defect are determined from the fine structure energies rather than from their centers of gravity. The corresponding relativistic quantum defect orbitals are different for each component of a multiplet and, if $c=0$, they retain the nodal structure of the large components of the hydrogenic Dirac wave function.

\section{Extension of RQDO method to E2 transitions}

Let us consider now electric multipole radiation. The operator responsible for this radiation is the electric $2^{\xi}$-pole moment, introduced by the general expression

$$
Q^{(\xi)}=e \sum r_{k}^{\xi}\left(\frac{4 \pi}{2 \xi+1}\right)^{1 / 2} Y_{\xi}^{\mu}
$$

Using the Racah tensor $C_{m}^{\xi}$, we have

$$
Q^{(\xi)}=e \sum r_{k}^{\xi} C_{m}^{(\xi)}
$$


In the particular case $\xi=2$ the transitions take place via electric quadrupole mechanism, E2. In this context, the electric quadrupole line strength for a transition between two states within the LSJ-coupling (which is the coupling scheme followed throughout this work) in the notation of the classical book by Condon and Shortley [16] is given by the equation

$$
S_{n l j, n^{\prime} l^{\prime} j^{\prime}}^{(2)}=\frac{2}{3}\left|\left\langle\alpha J\left\|Q^{(2)}\right\| \alpha^{\prime} J^{\prime}\right\rangle\right|^{2}
$$

where the matrix-elements have the form

$$
\left\langle\alpha J\left\|Q^{(2)}\right\| \alpha^{\prime} J^{\prime}\right\rangle=\sqrt{(2 J+1)\left(2 J^{\prime}+1\right)} W\left(S J L^{\prime} 2 ; L J^{\prime}\right)\left\langle\alpha L\left\|Q^{(2)}\right\| \alpha^{\prime} L^{\prime}\right\rangle \delta_{S S^{\prime}}
$$

where the Kronecker delta $\delta_{S S^{\prime}}$ appears because the electric $n$-pole operators do not depend on spin, and $W\left(S J L^{\prime} 2 ; L J^{\prime}\right)$ are the Racah W-coefficients, which can be described in terms of Wigner's 6-j symbols as

$$
W\left(S J L^{\prime} 2 ; L J^{\prime}\right)=(-1)^{S+J+L^{\prime}}\left\{\begin{array}{lll}
S & J & L \\
2 & L^{\prime} & J^{\prime}
\end{array}\right\} .
$$

Now, we define a line factor $R_{\text {line }}$ by

$$
R_{\text {line }}\left(S L J, S^{\prime} L^{\prime} J^{\prime}\right)=(2 J+1)^{1 / 2}\left(2 J^{\prime}+1\right)^{1 / 2} W\left(S J L^{\prime} 2 ; L J^{\prime}\right),
$$

and the line strength then becomes

$$
\begin{aligned}
S_{n l j, n^{\prime} l^{\prime} j^{\prime}}^{(2)} & =\frac{2}{3}(2 J+1)\left(2 J^{\prime}+1\right)\left(W\left(S J L^{\prime} 2 ; L J^{\prime}\right)\right)^{2}\left|\left\langle\alpha L\left\|Q^{(2)}\right\| \alpha^{\prime} L^{\prime}\right\rangle\right|^{2} \delta_{S S^{\prime}} \\
& =\frac{2}{3}\left(R_{\text {line }}\left(S L J, S^{\prime} L^{\prime} J^{\prime}\right)\right)^{2}\left|\left\langle\alpha L\left\|Q^{(2)}\right\| \alpha^{\prime} L^{\prime}\right\rangle\right|^{2} \delta_{S S^{\prime}} .
\end{aligned}
$$

It is important to stress that spin does not change during the transition because, as it was already mentioned, the relevant operator is spin-independent. The following selection rules apply to $\mathrm{E} 2$ transitions between LS-coupling states: $\Delta S=0$ and also $\Delta L=+2,+1,0,-1,-2$. But they do not apply neither from $L=0$ to $L=0$, nor from $L=1$ to $L=0$.

Therefore, the reduced matrix element $\left\langle\alpha L\left\|Q^{(2)}\right\| \alpha^{\prime} L^{\prime}\right\rangle$ provides the relative strength of different multiplets. We can write this reduced matrix element as the product of a single-electron reduced matrix element $\left\langle n L\left\|Q^{(2)}\right\| n^{\prime} L^{\prime}\right\rangle$, which depends only on the quantum numbers of the jumping electron, and a factor $R_{m u l t}$, that we shall call the multiplet factor, as follows:

$$
\left\langle\alpha L\left\|Q^{(2)}\right\| \alpha^{\prime} L^{\prime}\right\rangle=R_{\text {mult }}\left(\alpha L, \alpha^{\prime} L\right)\left\langle n L\left\|Q^{(2)}\right\| n^{\prime} L^{\prime}\right\rangle,
$$

where

$$
\left\langle n L\left\|Q^{(2)}\right\| n^{\prime} L^{\prime}\right\rangle=\left\langle L\left\|C^{(2)}\right\| L^{\prime}\right\rangle\left\langle R_{n l j}\left|Q^{(2)}\right| R_{n^{\prime} l^{\prime} j^{\prime}}\right\rangle
$$


In the last equation $\left\langle R_{n l j}\left|Q^{(2)}\right| R_{n^{\prime} l^{\prime} j^{\prime}}\right\rangle$ is the transition integral, $\left\langle L\left\|C^{(2)}\right\| L^{\prime}\right\rangle$ is the pertinent reduced matrix element, which can be evaluated using $3-\mathrm{j}$ symbols. The multiplet factor $R_{\text {mult }}$ may be expressed as follows:

$$
R_{\text {mult }}\left(\alpha L, \alpha^{\prime} L^{\prime}\right)=(2 L+1)^{1 / 2}\left(2 L^{\prime}+1\right)^{1 / 2} W\left(L_{c} L l^{\prime} 2 ; l L^{\prime}\right),
$$

the last symbol in Eq. (21) being the Racah W-coefficient

$$
W\left(L_{c} L l^{\prime} 2 ; l L^{\prime}\right)=(-1)^{L_{c}+L+l^{\prime}}\left\{\begin{array}{ccc}
L_{c} & L & l \\
2 & l^{\prime} & L^{\prime}
\end{array}\right\} .
$$

Finally, the line strength (18) takes the form (for $S=S^{\prime}$ ):

$$
S_{n l j, n^{\prime} l^{\prime} j^{\prime}}^{(2)}=\frac{2}{3}\left|R_{\text {line }}\left(S L J, S^{\prime} L^{\prime} J^{\prime}\right) R_{\text {mult }}\left(\alpha L, \alpha^{\prime} L^{\prime}\right)\left\langle L|| C^{(2)}|| L^{\prime}\right\rangle\left\langle R_{n l j}\left|Q^{(2)}\right| R_{n^{\prime} l^{\prime} j^{\prime}}\right\rangle\right|^{2} .
$$

The total line strength for a transition between multiplets is equal to the sum of the line strengths of all the multiplet lines:

$$
S\left(\gamma L, \gamma^{\prime} L^{\prime}\right)=\sum S\left(\gamma J, \gamma^{\prime} J^{\prime}\right)
$$

Thus, the line strength for a multiplet transition is

$$
\begin{aligned}
S\left(\gamma L, \gamma^{\prime} L^{\prime}\right) & =(2 S+1)\left|\left\langle\alpha L\left\|Q^{(2)}\right\| \alpha^{\prime} L^{\prime}\right\rangle\right|^{2} \\
& =(2 S+1)\left|R_{\text {mult }}\left(\alpha L, \alpha^{\prime} L^{\prime}\right)\left\langle n L\left\|Q^{(2)}\right\| n^{\prime} L^{\prime}\right\rangle\right|^{2}
\end{aligned}
$$

The relationships between the line strength $S^{(2)}$ (in atomic units, $e^{2} a_{o}^{4}$ ), the oscillator strength $f^{(2)}$ (dimensionless), and the transition probability $A^{(2)}\left(\right.$ in $s^{-1}$ ) for E2 transitions are given by [17]

$$
\begin{aligned}
& g^{\prime} A^{(2)}=\left(8 \pi^{2} \hbar \alpha / m \lambda^{2}\right) g f^{(2)}=\left(6.670310^{15} / \lambda^{2}\right) g f^{(2)}, \\
& g^{\prime} A^{(2)}=\left(32 \pi^{5} \alpha c a_{o}^{4} / 15 \lambda^{5}\right) S^{(2)}=\left(1.1199510^{18} / \lambda^{5}\right) S^{(2)},
\end{aligned}
$$

where $\lambda$ is the transition wavelength (in $\AA$ ), $g$ and $g^{\prime}$ are the degeneracies of the lower and upper state, respectively, and $\alpha$ is the fine-structure constant.

\section{The Z-expansion theory for E2 transitions}

Regularities in individual oscillator strengths along an isoelectronic sequence as functions of the nuclear charge have been predicted from conventional perturbation theory $[18,19]$, but these results can be extended to E2 transitions. Let us denote the transition integral

$$
\left|\left\langle R_{n l j}\left|r^{\xi}\right| R_{n^{\prime} l^{\prime} j^{\prime}}\right\rangle\right|=I_{n l j, n^{\prime} l^{\prime} j^{\prime}}^{(\xi)}
$$


Then, for a given operator, the variation of a matrix element as a function of $Z$ may be studied by the Rayleigh-Schrödinger perturbation theory: if we introduce $\rho=Z r$ and $\varepsilon=E Z^{-2}$ (in atomic units), the expansions of $\psi$ and $E$ in powers of the perturbation parameter $Z^{-1}$ are the following:

$$
\begin{aligned}
\psi_{n l j, n^{\prime} l^{\prime} j^{\prime}} & =\psi_{0}+\psi_{1} Z^{-1}+\psi_{2} Z^{-2}+\cdots \\
E & =Z^{2}\left(\varepsilon_{0}+\varepsilon_{1} Z^{-1}+\varepsilon_{2} Z^{-2}+\cdots\right) .
\end{aligned}
$$

In particular, the dipole transition integral $(\xi=1)$ is given by the $Z$-expansion

$$
\left|\left\langle\psi_{n l j}|\rho| \psi_{n^{\prime} l^{\prime} j^{\prime}}\right\rangle\right|=I_{n l j, n^{\prime} l^{\prime} j^{\prime}}^{(1)}=I_{0}^{(1)} Z^{-1}+I_{1}^{(1)} Z^{-2}+\cdots,
$$

where $I_{0}$ is the corresponding integral for hydrogen and the superscript (1) refers in all the cases to $\mathrm{E} 1$ or electric dipole transitions.

The dipole line strength, or squared radial integral Eq. (30), may be written as:

$$
S_{n l j, n^{\prime} l^{\prime} j^{\prime}}^{(1)}=S_{0}^{(1)} Z^{-2}+S_{1}^{(1)} Z^{-3}+S_{2}^{(1)} Z^{-4}+\cdots,
$$

and the expression for the E1 oscillator strength will be

$$
f_{n l j, n^{\prime} l^{\prime} j^{\prime}}^{(1)}=f_{0}^{(1)}+f_{1}^{(1)} Z^{-1}+f_{2}^{(1)} Z^{-2}+\cdots .
$$

For the line and oscillator strength, as well as for the transition probability $A$, it is possible to perform a paralell nuclear charge expansion representation in the quadrupole case to study systematic trends of $\mathrm{E} 2 S$ or $f$-values along an isoelectronic sequence. Here, the transition operator $\left(\rho^{2}\right)$ leads to [20]

$$
S_{n l j, n^{\prime} l^{\prime} j^{\prime}}^{(2)}=S_{0}^{(2)} Z^{-4}+S_{1}^{(2)} Z^{-5}+S_{2}^{(2)} Z^{-6}+\cdots
$$

with

$$
S_{0}^{(2)}=\left|\left\langle\psi_{n l j}\left|\rho^{2}\right| \psi_{n^{\prime} l^{\prime} j^{\prime}}\right\rangle\right|^{2} .
$$

For the transition probability $A$, the corresponding expansion is the following,

$$
A_{n^{\prime} l^{\prime} j^{\prime}, n l j}^{(2)}=A_{0}^{(2)} Z^{6}+A_{1}^{(2)} Z^{5}+A_{2}^{(2)} Z^{4}+A_{3}^{(2)} Z^{3}+A_{4}^{(2)} Z^{2}+\cdots,
$$

and the quadrupole oscillator strength may be written as

$$
f_{n l j, n^{\prime} l^{\prime} j^{\prime}}^{(2)}=f_{0}^{(2)} Z^{2}+f_{1}^{(2)} Z+f_{2}^{(2)}+f_{3}^{(2)} Z^{-1}+f_{4}^{(2)} Z^{-2}+\cdots
$$

where $f_{0}^{(2)}, f_{1}^{(2)}, f_{2}^{(2)}, \ldots$ are proportional to some power of $\Delta \varepsilon_{0}$, the hydrogenic transition energy corresponding to the transition under study. It is also very interesting to analyze the behaviour of $f^{(2)} Z^{-2}$ along the isoelectronic sequence. From Eq. (36) it follows that

$$
f_{n l j, n^{\prime} l^{\prime} j^{\prime}}^{(2)} Z^{-2}=f_{0}^{(2)}+f_{1}^{(2)} Z^{-1}+f_{2}^{(2)} Z^{-2}+\cdots
$$


When no change of the principal quantum number occurs during the transition, that is $\Delta n=0$, and if we ignore relativistic effects, $\Delta \varepsilon_{0}=0$, we have

$$
f_{l j, l^{\prime} j^{\prime}}^{(2)}=f_{3}^{(2)} Z^{-1}+f_{4}^{(2)} Z^{-2}+\cdots .
$$

In other words, the curve of $f^{(2)}$ versus $Z^{-1}$ would tend to an asymptotic zero value in the high- $Z$ side of the sequence. Eq. (38) is expected to be a good approximation to the behaviour of the oscillator strength with $Z^{-1}$ for E2 transitions at least for the first few ions of the sequence of a light element, e.g., $\mathrm{Na}$ I, where relativistic effects still are not very important. However, as soon as these effects set in, deviations from Eq. (38), as well as from all the expressions in this section, are expected to occur.

In this way, the dependence of the relativistic effects with the nuclear charge $Z$ in E2 transitions can be analysed by the calculation of the contribution of the relativistic effects (RE) in intra-configuration transitions, which can be done by comparing the results from the calculations performed with the non-relativistic (QDO) and the relativistic (RQDO) formulations of the Quantum Defect Orbital method. The weighting of the relativistic effects on the oscillator strengths can be measured in the following manner:

$$
R E=\left[\frac{f_{Q D O}-f_{R Q D O}}{f_{Q D O}}\right] \cdot 100 .
$$

Relativistic effects lead to a decrease in the magnitude of the oscillator strengths of the ions under study, that is, $f_{R Q D O}$ is generally found to be smaller than $f_{Q D O}$ for a given E2 transition. We have analysed this effect for the electric quadrupole transitions in the Na sequence as a function of the nuclear charge $Z$, and fitted the RE-value individually for each of the fine-structure transitions to a polynomic function of $Z$. The fitting formulae obtained for the $n l j \rightarrow n l j^{\prime}$ E2-lines has the following general expression

$$
R E_{n l j \rightarrow n l j^{\prime}}=a+b Z+c Z^{2} .
$$

These equations can be found in the paper of Charro et al for Na-like ions [21], and are plotted in Figure 1. It is apparent that the influence of the relativistic effects in the oscillator strengths for all the fine-structure transitions decreases as $n$ increases. It is also found that the relativistic effects appear to be generally less important as $\ell$ increases for a given $n$. These two features were to be expected, in the presently analysed range of ions, where the largest atomic number is $Z=36$. Hence, the dominant relativistic effects are those of direct character, which are appropriately included in the RQDO procedure. 
Fig. 1 Contribution of the relativistic effects in intraconfigurational fine-structure E2 transitions of Na-like ions.

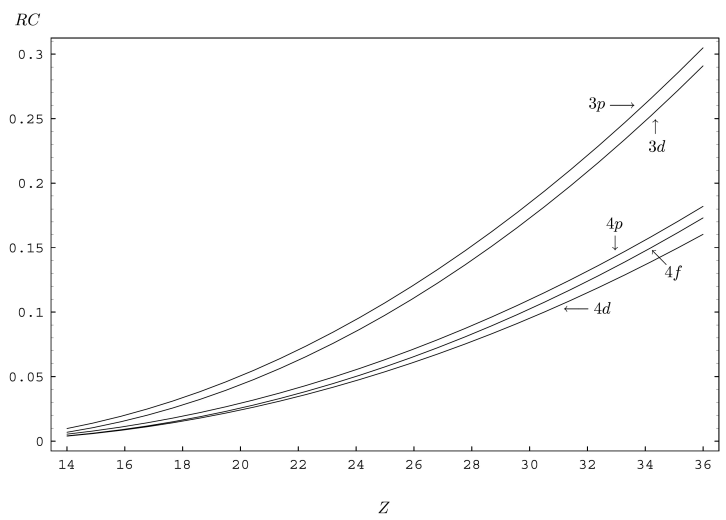

\section{Atomic data and UV and X-ray atomic spectra for ions in fusion plasma}

Given that the regular behaviour of atomic data as oscillator strengths along isoelectronic sequences has proven to be a useful tool for analysing a large body of $f$ values, the analysis of the systematic trend of the $A$-values for E2 transitions along isoelectronic sequences was carried out in several previous works using the RQDO method, both for allowed and forbidden transitions. This study may also be exploited to obtain additional oscillator strengths by simple interpolation techniques. From the analysis of systematic trends for E2 transitions, several calculations have been performed, in particular the following isoelectronic sequences:

- B-sequence: $Z=37-82$ (see Ref. [22]).

- $\mathrm{K}$-sequence $\mathrm{Z}=25$ to $\mathrm{Z}=80$ (see Ref. [23]).

- Al-sequence (see Ref. [24]).

- Ga-sequence (see Ref. [25]).

- Na-sequence. E2 transitions (see Refs. [21, 26]).

As an example, the behaviour of E2 transition probabilities along the boron isoelectronic sequence is graphically analysed in Figure 2. The value of $\log A$ has been plotted against the atomic number $Z$. The available comparative data have also been included [27, 28]. This figure is useful for two purposes: the first one is to show the agreement or deviations among the different sets of data, the second one is to reflect the systematic trends obeyed by the individual RQDO $A$-values along the isoelectronic sequence, which have long been considered as a qualitative proof of correctness, and can be used for the interpolation or extrapolation of non-calculated data. Inspection of Figure 2 reveals a rather good general agreeement between our results and the comparative data.

The interest in line emission from highly-ionised atoms in tokamak devices is, in the first instance, due to the effect of impurities on the overall performance of the tokamak as a fusion device. The most common impurity elements are $\mathrm{Ti}, \mathrm{Cr}, \mathrm{Fe}, \mathrm{Ni}$, 
Fig. 2 Transition Probabilities for E2 forbidden lines in boron isoelectronic sequence, being the comparative data reported by Froese Fischer [27] for (b) and (c), and by Cheng et al [28] for (d).

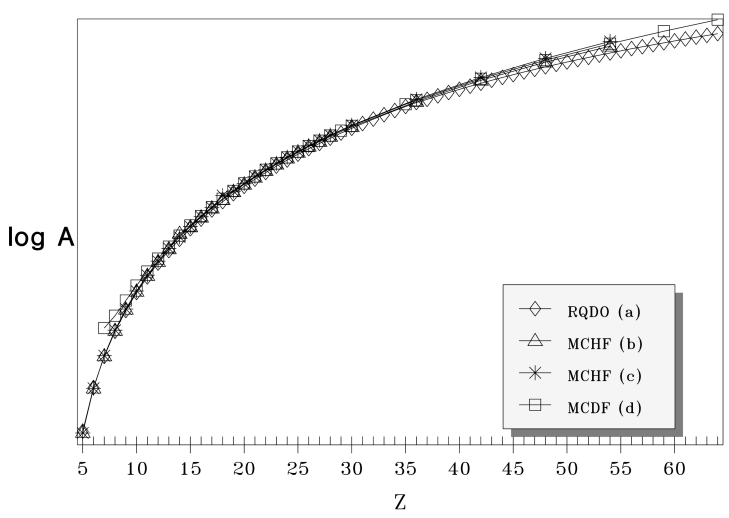

the lighter $\mathrm{C}, \mathrm{N}, \mathrm{O}$, and the rare gases $\mathrm{Ne}$ and $\mathrm{Ar}$ [29]. Allowed transitions in ions of metals as $\mathrm{Zr}$ have been reported using RQDO method [30], but also interesting in tokamaks are the forbidden lines, which are valuable diagnostic monitors of the ion motion, and of the metal impurity concentrations. For E2 transitions several calculations using this semiempirical method have been performed in order to predict the UV and X-ray spectra for several metals, in particular highly-charged ions as is the case of Ti XII and FeXVI (Figures 3 and 4). We have done a simulation of the E2 spectrum of these two ions, which include only the intensities of the RQDO E2 transitions [31].

Fig. 3 The simulated spectra for Ti XII according to RQDO calculations for forbidden (E2) transitions.

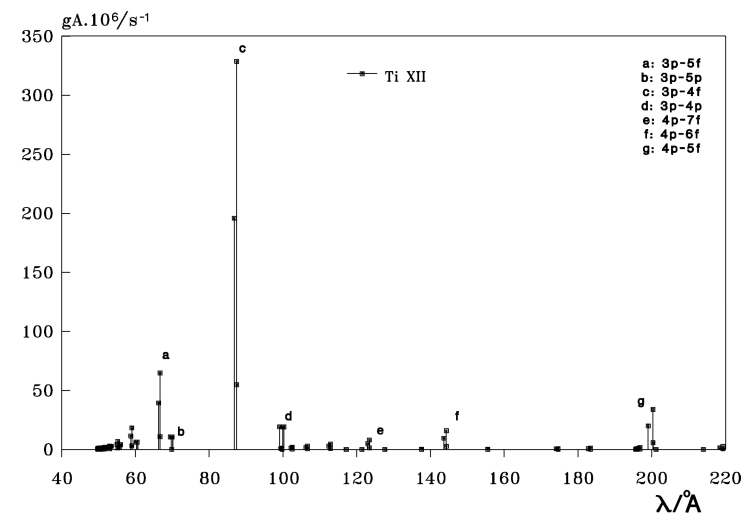


Fig. 4 The simulated spectra for Fe XVI according to RQDO calculations.

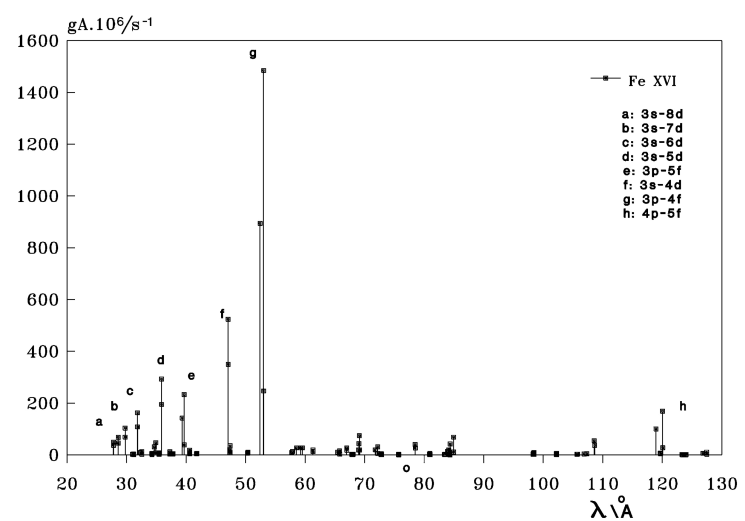

\section{Concluding remarks}

The RQDO formalism, as opposed to sophisticated and costly self-consistent-field procedures, is a simple but reliable analytical method based on a model Hamiltonian. It has the great advantage of the computational effort not being increased as the atomic system dealt with becomes heavier and is capable of achieving a good balance between computational effort and accuracy of results. The method is a useful tool for calculating UV and X-ray atomic spectra for ions and transitions which are difficult to evaluate, and may play an important role in the future, when fusion becomes a reality.

Acknowledgements Financial support from Spanish MINECO (MTM2014-57129-C2-1-P) and Junta de Castilla y León (VA057U16) is acknowledged.

\section{References}

1. Glasser M.L., March N.H., Nieto L.M.: Proposed interpretation of the transverse magnetic field dependence of the melting temperature $T_{m}(B)$ of a two-dimensional one-component plasma driven by logarithmic interactions. Phase Transitions 85, 10181021 (2012)

2. March N.H., Capuzzi P., Tosi M.P.: Changes of magnetization and entropy across the melting curve for a classical two-dimensional plasma in a magnetic field. Phys. Lett. 327, 226-229 (2004)

3. March N.H., Tosi M.P.: Diffraction and transport in dense plasmas: Especially liquid metals. Laser Part. Beams 16, 71-81 (1998)

4. March N.H., Tosi M.P.: Thermal conduction in strongly coupled plasmas: Especially liquid alkali metals. J. Plasma Phys. 57, 121-127 (1997)

5. Amato M.A., March N.H.: Structure and equation of state of metallic plasmas. Laser Part. Beams 14, 685-697 (1996)

6. Robson R.E., Paranjape B.V., March N.H.: Haeffner effect in a binary isotopic dilute plasma Plasma Phys. Control. Fusion 36, 635-640 (1994) 
7. Amovilli C., March N.H., Pfalzner S.: Model of confined atoms in arbitrary static electricfields. Relevance to nondegenerate plasmas. Phys. Chem. Liq. 24, 79-89 (1991)

8. Mahanty J., March N.H., Paranjape B.V.: Interaction between rare-gas atoms outside metalsurfaces. Role of plasma excitations. Appl. Surf. Sci. 33, 309-314 (1988)

9. Leung W.B., March N.H.: Resistivity of a structureless Hydrogen plasma due to weak electron-ion interaction. Plasma Phys. Control. Fusion 19, 277-281 (1977)

10. Stratton B.C., Bitter M., Hill K.W., Hillis D.L., Hogan J.T.: Passive Spectroscopic Diagnostics for Magnetically Confined Fusion Plasmas. Fusion Sci. Techn. 53, 431-486 (2008)

11. Jupén C., Denne-Hinnov B., Martinson I., Curtis L.J.: Additions to the Spectra of Highly Ionized Molybdenum, Mo XXIVMo XXVIII. Phys. Scripta 68, 230-232 (2003)

12. Karwowski J., Martín I.: Quasirelativistic formulation of the quantum-defect-orbital method. Phys. Rev. A 43, 4832-4836 (1991)

13. Martín I., Karwowski J.: Quantum defect orbitals and the Dirac second-order equation. J. Phys. B: At. Mol. Phys. 24, 1539-1542 (1991)

14. Charro E., Martín I.: Relativistic effects and sExtension of the RQDO Method to study forbidden transitions, in "Foundations of Quantum Physics". Anales de Física. Monografías. R. Blanco et al. (Eds.). Real Sociedad Española de Física, Madrid (2002), 331-349

15. Martín I., Karwowski J., Bielińska-Waz D.: Matrix elements of $r^{q}$ in relativistic quantum defect orbital theory. J. Phys. A: Math. Gen. 33, 823-830 (2000)

16. Condon E.U and Shortley G.H. The Theory of Atomic Spectra. Cambridge University Press, Cambridge (1935)

17. Sobelman I.I.: Atomic Spectra and Radiative Transitions. Springer-Verlag, Berlin (1979)

18. Cohen M., Dalgarno A.: An Expansion Method for Calculating Atomic Properties. VIII. Transitions in the Hartree-Fock Approximation. Proc. Roy. Soc. A 293, 359-364 (1966)

19. Dalgarno A., Parkinson E.M.: An Expansion Method for Calculating Atomic Properties. X. $1 s^{21} S-1 s n p^{1} P$ Transitions of the Helium Sequence. Proc. Roy. Soc. A 301, 253-260 (1967)

20. Biémont E., Godefroid M.: Hypervirial Theorem, Screening Parameters and Electric Quadrupole Oscillator Strengths in the Sodium Sequence. Phys. Scr. 18, 323-331 (1978)

21. Charro E., Martín I.: Relativistic effects and systematic trends in electric quadrupole transition probabilities for Na-like ions. J. Phys. B: At. Mol. Phys. 35, 3227-3241 (2002)

22. Charro E., López-Ferrero S., Martín I.: Forbidden emission coefficients for intraconfiguration transitions $2 p_{3 / 2} \rightarrow 2 p_{1 / 2}$ along the boron sequence. J. Phys. B: At. Mol. Phys. 34, 4243-4257 (2001)

23. Charro E., Curiel Z., Martín I.: Atomic data for M1 and E2 emission lines in the potassium isoelectronic sequence. A \& A 387, 1146-1152 (2002)

24. Charro E., López-Ferrero S., Martín I.: Trends in E2 and M1 transition rates between $3 p_{3 / 2}$ and $3 p_{1 / 2}$ levels in $3 s^{2} 3 p^{k}$ systems. A \& A 406, 741-749 (2003)

25. Charro E., Curiel Z., Martín I.: Spontaneous radiative decay rates in Ga-like ions. Int. J. Quantum Chem. 108, 744-753 (2008)

26. Charro E., Martín I.: Systematic Trends in E2 Transitions along the Sodium Isoelectronic Sequence. Int. J. Quantum Chem. 90, 403-409 (2002)

27. Froese Fischer C.: Multiconfiguration Hartree-Fock Breit-Pauli results for ${ }^{2} P_{1 / 2}-{ }^{2} P_{3 / 2}$ transitions in the boron sequence. J. Phys. B: At. Mol. Phys. 16, 157-165 (1983)

28. Cheng K.T., Kim Y.-K., Desclaux J.P.: Electric dipole, quadrupole, and magnetic dipole transition probabilities of ions isoelectronic to the first-row atoms, Li through F. At. Data Nucl. Data Tables 24, 111-189 (1979)

29. Peacock, N.J., Stamp, M.F., Silver, J.D.: Highly-Ionized Atoms in Fusion Research Plasmas. Phys. Scr. T8, 10-20 (1984)

30. Charro E., López-Ayuso J.L., Martín I.: Oscillator strengths for spectral lines of Zr III of astrophysical importance. J. Phys. B: At. Mol. Phys. 32, 4555-4565 (1999)

31. Charro E., Martín I.: Spectroscopy of forbidden transitions in ions of astrophysical interest. J. Mol. Struct. (Theochem) 621, 75-85 (2003) 